\title{
Evolutionarily Stable Dispersal Strategies
}

\author{
Hugh N. Comins \\ Environmental Biology Dept., R.S.B.S., Australian National \\ University, Canberra 2600, Australia
}

William D. Hamilton
M useum of Zoology, U niversity of Michigan, Ann Arbor, Michigan 48109, U.S.A.

AND

ROBERT M. MAY

\section{Biology Department, Princeton University, Princeton, N.J. 08540, U.S.A.}

(Received 16 April 1979, and in revised form 20 April 1979)

\begin{abstract}
Using the idea that life-history parameters are subject to natural selection and should approach values that are stable optima, with the population immune to invasion by mutant individuals, we derive an analytic expression for the evolutionarily stable dispersal rate in a stochastic island model with random site extinction. The results provide interesting contrasts between three different optimization criteria: species survival, individual fitness and gene fitness. We also consider the effects of sexual reproduction, and of localized migration (stepping-stone structure).
\end{abstract}

\section{Introduction}

Movement leading to dispersal is an essential property of living things. Dispersal enables a species to extend its range, and reduces the chance of extinction due to local fluctuations (including such fluctuations as might result from excessive local population pressure and exploitation of resources). However, notwithstanding the undoubted benefits to the species (and indeed to the stability of the ecosystem), it is by no means obvious what advantage an individual organism gains by undertaking a perilous dispersal movement instead of staying back to compete more safely in the locality where it was reared.

In a simulation study using a "stepping stone" model (Wright, 1969) of population structure, Roff (1974) showed that the dispersal strategy which 205 
maximizes site occupancy, and is thus to be regarded as optimal from the species point of view (May, 1974), differs significantly from that produced by genetic selection. Thus evolution will lead to the species exploiting less of its habitat than it conceivably might, a result of considerable ecological interest.

The determination of the evolutionarily stable dispersal strategy (ESS) by simulation is by nature a very lengthy process, particularly when stochastic elements are present. Thus the investigation of how the ESS varies with different chances of surviving migration and differing degrees of environmental variability would be greatly facilitated if the ESS could be obtained analytically. Hamilton and May (1977) made a start in this direction and obtained results for a model which had a simple stochastic pattern. The present paper extends their results to transient environments and general probability distributions for the number of progeny, and allows more than one organism to occupy each site.

In addition to the main result, which is in qualitative agreement with Roff's model, the effects of two other features of the simulation model are considered in less detail. These are sexual reproduction and stepping stone (as opposed to island model) migration. Both these additions reduce the ESS migration rate, although the affect of sexual reproduction is usually small.

The full mathematical derivation of the ESS migration rate is given in Appendix A. However, the important points are repeated in the text, so that the appendices need only be used for reference.

\section{The Model}

The basic model (see Fig. 1 and Table 1) is essentially a discrete generation island model (Wright, 1969) in which the processes of reproduction, migration and competition are stochasticized. Because of the stochastic effects it is possible for a site to become empty if all offspring in the site happen to emigrate and there are no immigrants. In addition a degree of environmental variability will be introduced in the form of a fixed probability $\boldsymbol{X}$ that a site is destroyed by exogenous forces during a onegeneration time interval. As in Hamilton \& May's (1977) model reproduction is assumed to be asexual, and individuals of each genotype have a certain probability of being born with a predisposition to migrate.

Suppose that there are a very large number of sites each capable of supporting $k$ reproductive adults. Each adult independently produces a number of offspring according to a probability distribution which is independent of genotype. The mean number of offspring per adult is $\tau$, giving an average total of $n=k \tau$ off spring per site. Each of these offspring is independently determined to be migratory with probability $v$ or sedentary 


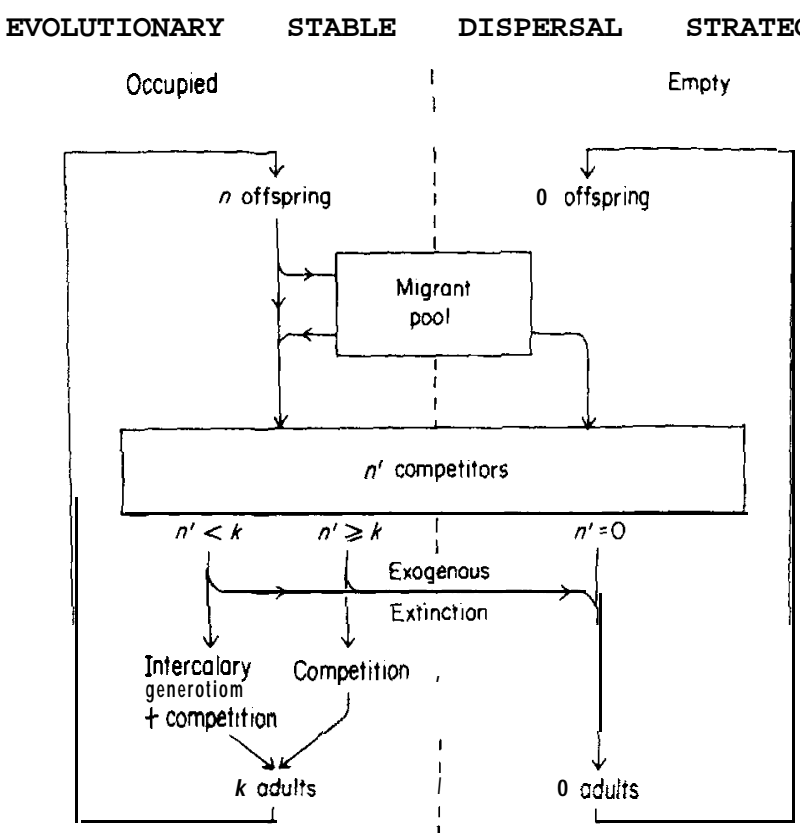

Fig. 1. Cycle of events in each generation.

with probability I $-v$, where $\mathrm{v}$ depends on the genotype. Migrants enter a pool from all sites, where a proportion $1-p$ is removed (hence $p$ is the survival rate for migration). The survivors are then redistributed among the sites; the number of a given genotype arriving at a given site is assumed to be Poisson distributed, with the mean for each type equal to the number of that type in the pool divided by the total number of sites.

\section{TABLE 1}

\section{Parameters and variables in the mode}

\begin{tabular}{ll}
\hline$k$ & number of adults per site \\
$\tau$ & average number of offspring per adult \\
$n$ & average number of offspring per site $=k \boldsymbol{\tau}$ \\
$v$ & probability of migration \\
$\boldsymbol{X}$ & exogenous extinction rate \\
$p$ & chance of surviving migration \\
$f$ & proportion of sites occupied \\
$\rho$ & mutant gene frequency \\
$F$ & equilibrium variance parameter \\
$\mathrm{C}$ & ratio of destroyed sites to remaining sites \\
$\boldsymbol{\theta}$ & $\begin{array}{l}\boldsymbol{X} \boldsymbol{X} /(1-\boldsymbol{X}) \\
\text { product of } \boldsymbol{p} \text { and } \mathbf{f}\end{array}$ \\
\hline
\end{tabular}


Sedentary local offspring and immigrants compete for each site in such a manner that by the time of the next generation there are exactly $k$ reproducing adults. If there are more than $k$ competitors the excess must die or fail to reproduce. If there are fewer than $\mathbf{k}$ competitors than we require intercalary non-migratory generations in order to fill the site. This assumption of fixed site size may seem somewhat artificial but it is necessary to make the analysis tractable; at the same time, bearing in mind possibilities of growth and vegetative reproduction, it is not very unreasonable. In either case we assume fair competition, so that each of the $k$ adults has the same chance of being descended from each of the original competitors. This represents an approximation unless the number of competitors is much greater than $\mathbf{k}$. The case where immigrants have a systematically lower chance of surviving competition can be incorporated as an additional effective mortality, decreasing $p$.

In summary, this model extends that of Hamilton and May in having the number of adults $k$ at each site allowed to be more than one, and in having a degree of environmental variability due to the exogenous extinction rate $\mathrm{X}$. The extinction of sites can be regarded either as a random process, or as an aging process with type II "survivorshop" (i.e., probability of death independent of age). This is reminiscent of a feature of the earlier model which gave to the adult organism occupying a site a certain probability of dying; however in the current model it is as if the site itself disappears; or, if the site is considered to persist while occupants die within it, then all are assumed to die together. Disappearance of the old sites is assumed to be balanced by the creation of an equal number of new empty sites; these can only be colonized by migrants, so it is at once evident that large values of $\mathrm{X}$ make migration more attractive.

At this stage of its development our model differs from Roff's simulation model most significantly in the assumptions of asexual reproduction and island model migration (as opposed to Roff's 6 × 6 stepping stone model).

\section{Evolutionarily Stable Strategy (ESS)}

It is assumed that migration rate is a continuous genetic character. An evolutionarily stable state in terms of such a character can consist of either a single phenotype or a mix of phenotypes at specific frequencies with the characteristic that all genes coding for small changes in the migration rate of a phenotype are disadvantageous. In addition to this genetic stability criterion, which must be satisfied for every phenotype, the stability of a polymorphism requires that there be frequency dependent selection to stabilize the relative frequency of each phenotype. 
The possibility of polymorphism in the ESS will not be pursued in this paper; in the remainder of this paper it will be assumed that the ESS is a single phenotype. The observational difference between a polymorphism and a single phenotype having the same average migration rate is an increased correlation between migration tendencies of offspring of the same parent. Such a correlation could conceivably contribute to stability in a way that would exclude invasion by a single "average" type. However, a polymorphism cannot exist unless all the component phenotypes are stable with respect to small changes in migration rate. In the case of a single phenotype only one ESS is ever found, representing a compromise between colonizing ability and risk of loss of the home site. It is therefore hard to see how two or more phenotypes could simultaneously be stable, so that a polymorphism seems rather unlikely, although the possibility has not been entirely ruled out.

In order to derive the ESS dispersal rate we thus assume that there are two types of individuals present; the "wild-type" use the putative ESS dispersal rate $v$ while those bearing the mutant gene have a slightly different dispersal rate $\mathrm{v}+\boldsymbol{\varepsilon}$. We must then find $v$ such that the mutants are at a disadvantage for any small $\varepsilon$, whether positive or negative. That is, the frequency of mutant genes $\rho^{\prime}$ in the next generation must be less than the current frequency $\rho$ (see Table 1). Since $\boldsymbol{\rho}^{\prime}-\boldsymbol{\rho}$ has a term linear in $\boldsymbol{\varepsilon}$, a necessary condition for an ESS is stationarity, that is the derivative of $\rho^{\prime}-\rho$ with respect of $v$ must be zero.

The complete description of the state of the system requires $k+2$ parameters; the proportion of sites which are occupied $f$, and $k+1$ fractions $\phi_{0}, \phi_{1}, \ldots, \phi_{k}$, where $\phi_{i}$ is the fraction of occupied sites in which $i$ out of the $k$ adults are mutants. Since $\phi_{0}+\ldots+\phi_{k}=1$, the $\phi$ 's can be regarded as the probability distribution of $i / k$ in occupied sites. Fortunately the ESS can be determined without knowing this full distribution, since the ESS condition only depends on $f$, and the mean $\rho$ and equilibrium variance $S$ of $i / k$, It can be shown, as in Wright's island model (1969), that (ignoring terms in $\boldsymbol{\varepsilon}$ ) the site occupancy fraction $f^{\prime}$, the mean mutant frequency $\rho^{\prime}$ and the mutant frequency variance $S^{\prime}$ for the next generation can all be obtained from the current $f, \rho$, and $S$ without reference to the rest of the distribution. For conformity with Wright's (1969) notation we will express the variance in terms of the gene frequency independent parameter $F=S /(\rho(1-\rho))$; it will turn out that the state of the system is described adequately by the three parameters $f, \rho$ and $F$.

Both $f$ and $F$ rapidly approach equilibria which are independent of $\rho$ except for terms of order $\boldsymbol{\varepsilon}$. The equilibrium value off is given by the implicit equation [see Table 1 and equation (A3)]: 


$$
\mathbf{f}=\left[\frac{C+\mathrm{e}^{-n(1-v)} \mathrm{e}^{-n v p f}}{1-\mathrm{e}^{-n v p f}}+1\right]^{-1}
$$

where $\mathrm{C}=X /(1-\mathrm{X})$. Although appendix A derives the ESS for a general distribution of family size, we will only consider here the case where the number of offspring is Poisson distributed. The equilibrium value of the variance parameter is then given by

$$
F=\frac{1-(1-X)\left(1-k^{-1}\right)\left[H(n v \theta / \alpha)+\left(f^{-1}-1\right) H(n v \theta)\right]}{1-(1-X)\left(1-k^{-1}\right)(1-\alpha)^{2} H(n v \theta / \alpha)}
$$

where $\alpha=v \theta /[(1-v)+v \theta], \theta=p f$, and the sigmoid-shaped function $H(a)$ is defined in (A44). This function has the series expansion $\boldsymbol{H}(\boldsymbol{a})=$ $\mathrm{e}^{-a} \sum_{i>1}\left[(i-1) a^{i} / i i !\right]$, but can be evaluated more efficiently using standard techniques for exponential integrals. Note that if $k=1$ we obtain the simple result $F=1$ as expected. The ESS condition (for Poisson distributed offspring) is found to be

$$
C+\{1-n v(1-\theta)\} Z=[(\alpha / \theta)(1-Z)-n v Z][(1-\theta)-(1-\alpha) F]
$$

where $\mathrm{Z}=\exp [-n\{1-v+v \theta\}]$. These equations can be solved as follows for given $p, X$ and $n$. Select a value of $v$; calculate $f$ by solving (1) (the result is unique). Provided the results are feasible, determine $F$ from (3) and then $k$ from (2). Invert the graph of $\mathbf{k}$ as a function of $v$ to obtain the ESS migration rate as a function of number of adults per site $k$. Since the graphs of $k$ against $v$ are invariably monotonic decreasing there is only one ESS for any $p, \boldsymbol{X}, n$, and $\mathrm{k}$.

\section{ESS Results}

Figures 2-5 show some typical results for the ESS dispersal rate given by equations (1)-(3). Note that when the number of adults per site $\mathbf{k}$ varies in these graphs, it is the total expected offspring $n$ which is kept constant, rather than the average offspring per adult $\tau$, since this is much more convenient for calculation. If however we wish to take the limit of very large site size $\mathbf{k} \rightarrow \infty$ then it is only reasonable to let the expected total offspring $\boldsymbol{n}$ become large as well and in referring to the limit $k \rightarrow \infty$ we shall assume that this happens.

All the graphs have the property that the ESS migration rate decreases as the number of adults per site is increased. It also decreases whenever the total number of offspring increases. At the same time the ESS migration rate always remains above a certain threshold $v$ at which non-migration ceases to offer the individual offspring the best option for becoming an established 


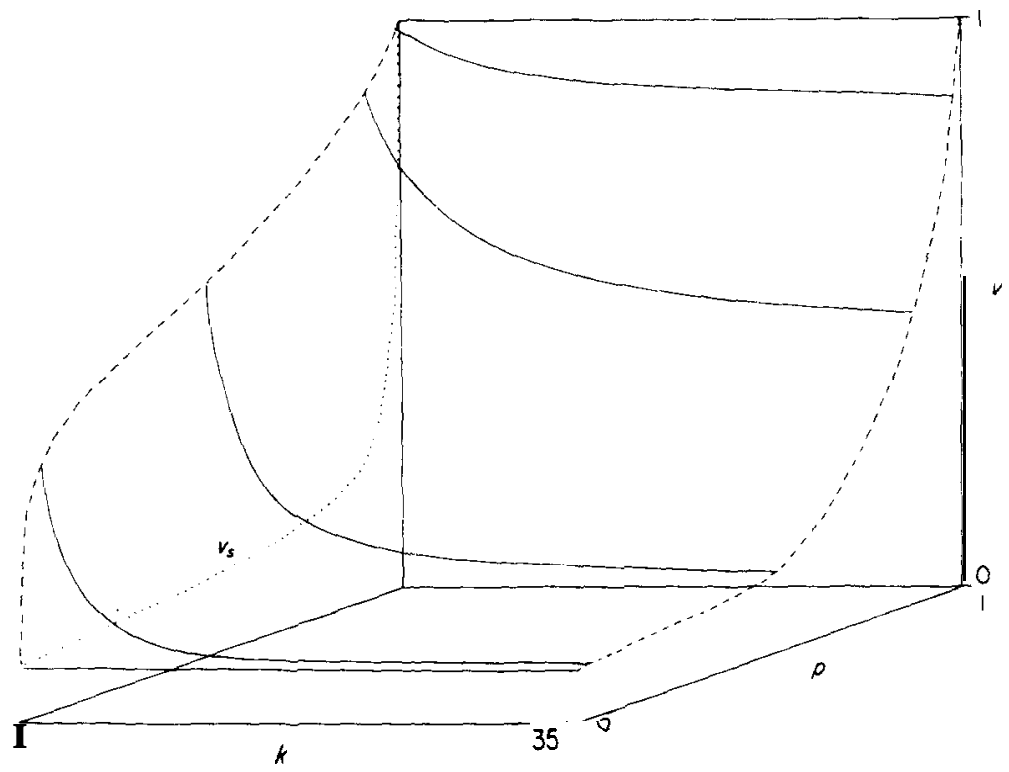

FIG. 2. 3-dimensional plot of the ESS migration probability $v$ as a function of site size $k$ and probability $\mathrm{p}$ of surviving migration. The average number of offspring per site is $\mathrm{n}=10$ and there is no exogeneous extinction. In the $k=1$ plane we show the species optimal migration probability $v_{s}$ as a function of migration survival $p$.

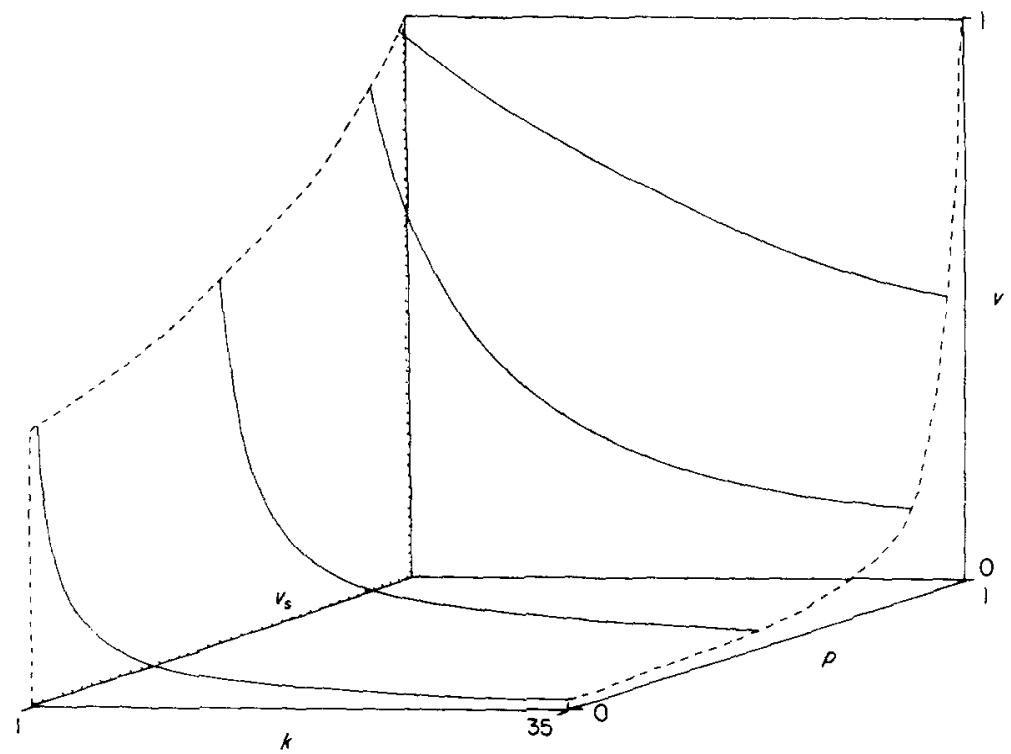

FIG. 3. As Fig. 2, but with average number of offspring per site $n=500$ 


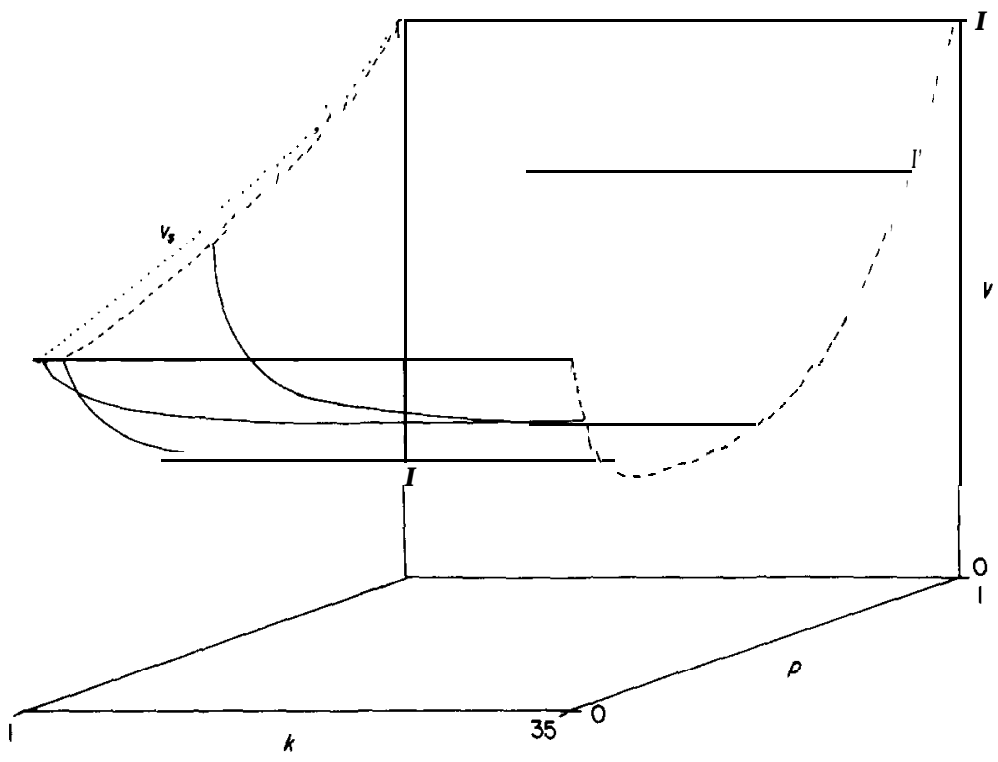

FIG. 4. As Fig. 2, but with $10 \%$ exogenous extinction $(\mathrm{X}=0-1)$.

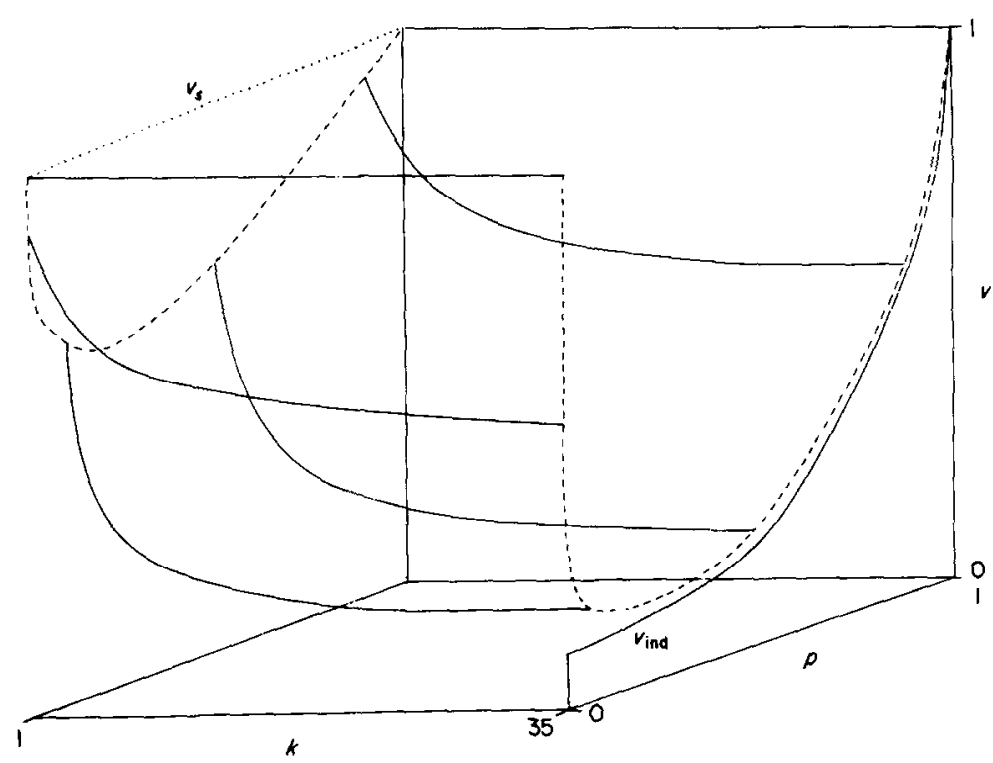

FIG. 5. As Fig. 2, but with $10 \%$ exogenous extinction $(X=0 \cdot 1)$ and average number of offspring per site $\boldsymbol{n}=500$. An additional graph in the $\boldsymbol{k}=35$ plane shows the individual optimum migration probability $\boldsymbol{v}_{\text {IND }}$ as a function of migration survival $\boldsymbol{p}$. 
adult. This threshold $v$, which we symbolize $v_{\text {IND }}$, merits further explanation. If a pre-dispersal offspring has a view only to maximizing its chance of becoming a reproductive adult, and if both in its group and in the population at large the migration rate is $v$, then its chance is maximized by migrating if $v<v_{\mathrm{IND}}$, by not migrating if $v>v_{\mathrm{IND}}$, while if $v=v_{\mathrm{IND}}$ chances either way are equal. Thus if the individual's short term selfish view were representative of natural selection, $v_{\text {IND }}$ would establish as the ESS. It hardly need be said that the suggested view is too limited; to look no further ahead than immediate adulthood is a very inadequate evolutionary viewpoint. This is particularly obvious in the present asexual model where long term individual and genic viewpoints actually coincide. Apart from demonstrating the inadequacy of this apparently plausible but simplistic view, the evaluation of $v_{\text {IND }}$ provides a true lower limit to which the ESS rate must converge as $\mathbf{k} \rightarrow \infty$, as explained below. In this limit it is easily shown that

$$
v_{\mathrm{IND}}=\frac{\mathrm{X}}{1-(1-X) p} \text {. }
$$

This equation represents a balance between the chance of establishing in the home site and the overall value of migrating. The latter depends on the frequencies of the three possible outcomes: dying en route, arriving at another occupied site, and finding an empty site. Since the competition at another occupied site is exactly the same as at the home site, only the third outcome can be considered favorable. It may indeed be verified that if $\mathrm{X}=0$ (giving no empty sites when $\mathbf{k} \rightarrow$ co) then $v_{\text {IND }}=0$ unless migrants incur no additional mortality $(p=1)$.

An example of (4) with $X=0 \cdot 1$ is plotted in Fig. 5 and placed so as to indicate the limit towards which the ESS rate (dashed curve) is falling as $k$ increases.

There are two ways of understanding the decline in the ESS with increasing numbers of adults or number of offspring, and also the convergence just mentioned. From the point of view of a gene, migration has the advantage of allowing competition for sites at which it is under-represented. Clearly this will only be worthwhile if there is likely to be a significant difference in gene frequencies between the starting and end points of the migration. This is the case when $k$ and $n$ are small, but as $k \rightarrow \infty, n \rightarrow$ co the genetic variance between sites declines to zero, so that there is no longer a difference between occupied sites from the gene's point of view. This now coincides with the selfish individual point of view expressed by (4), in which the only advantage of migration results from the possibility of colonizing an extinct site.

The second way of regarding this phenomenon is in terms of inclusive fitness. A particular offspring is genetically identical to about $\mathbf{I} / \mathbf{k}$ of the 
other offspring at its site, and is unrelated to the others. Thus its tendency to migrate when this is not individually favorable should be smaller for large $k$. The equivalence of these approaches to problems of selection in grouped populations is explained for a more elementary example in Hamilton (1975).

The ESS can also be compared with the optimum migration rate for the species, that is the value $v_{s}$ which maximizes the proportion of sites occupied. A qualitative difference between Hamilton and May's (1977) results and Roff's is that in the former the ESS migration rates are always found to be greater than the species optimum, whereas Roff always finds the ESS to be smaller.

A comparison of the migration rates for maximum site occupation plotted in the $k=1$ planes of Figs 2-5 shows that for a large total number of offspring (as in Roff's model) the maximum occupation $v$ is extremely sensitive to Hamilton \& May's (1977) assumption of no exogenous extinction $(X=0)$. Roff's model, on the other hand, includes environmental variations, which have a similar effect to $X>0$ in making migration more rewarding. Thus the differences in the two earlier models can be understood from the abrupt change found in the present model as X departs from a value of zero.

Another unexpected feature of the model for $X>0$ is related to this effect. With $X>0$ the ESS surfaces curve sharply upward for low migration survival $p$. The idea of increasing migration rate when the chance of surviving decreases is at first sight paradoxical. It suggests however that the first priority for low $p$ is to maintain a certain level of colonization of empty sites, and that competition for the home site is being left to any surplus offspring. This effect only occurs when the species is hard-pressed to maintain itself; in the extreme case only one migration rate can ensure continuance-applying both for individual lines and for species. It is clear that the ESS and species optimum $v$ must coincide in this case. Figures 4 and 5 show that the "width" of the high ESS "rim" (i.e., roughly the distance of this upturning portion of the surface from the plane $p=0$ ) decreases with $n$, tending to zero as $n \rightarrow \infty$. This is understandable if we note that for a non-stochastic version of the model the species just maintains itself if $n p>1 /(1-X)$; hence it is to be expected that the width will be inversely proportional to $n$.

\section{Additional Effects}

It is possible to infer the effect on the ESS of two additional considerations which cannot be included in the full model; sexual reproduction and stepping-stone migration. 
Appendix $\mathrm{C}$ derives the ESS for hermaphroditic sexually reproducing organisms in the limit of large total number of offspring $n$. The analysis proceeds in much the same way as before except that the $\mathbf{k}$ adult organisms are replaced by $\mathbf{2 k}$ genes. The result is surprisingly simple: the ESS for sexual reproduction with site-size $\mathbf{k}$ is obtained by substituting $k+\frac{1}{2}$ for $\boldsymbol{k}$ in the ESS formula for asexual reproduction with site-size $k$. Thus sexual reproduction effectively increases the site size by $\frac{1}{2}$. This is only important if $\mathbf{k}$ is very small. This simple result is not expected to extend to the stochastic case; however the relative importance of sexual reproduction is presumably no greater.

Introducing stepping-stone migration (Kimura \& Weiss, 1964) has the effect of producing local correlations in gene frequencies. Thus the endpoint of migration is more likely to have a similar gene frequency to the starting point. As seen in the last section, this makes migration less worthwhile from the gene point of view, so the ESS $v$ should be closer to the individual optimum. The change to a stepping stone model seems also to be of minor importance. A derivation of this result (for the case of no exogenous extinction, $X=0$ ) will be published elsewhere (Comins, 1980).

\section{Discussion}

From the gene or "inclusive fitness" point of view, there are two advantages in having a high dispersal rate. The first advantage is the colonization of empty sites, which also aids individual emigrants by enhancing their reproductive potential. The second advantage is the opportunity to compete for other sites in which the migrator allele is under-represented. Merely changing one arena of competition for another where numbers are the same is clearly of no advantage to an individual emigrant, and making the change may entail considerable risk. Nevertheless this "recolonization" effect is important on the genetic level, as can be seen from the extreme case of a completely sedentary phenotype in an environment where no sites are ever empty (Hamilton \& May, 1977). In such a case there is never any advantage to individual emigrants; yet still at the ESS much migration may occur.

The chance that a migrant entering a non-empty site finds a significantly different gene frequency clearly depends on the equilibrium variance in gene frequency. If the number of adults per site and the number of offspring per site are very large, all sites tend to approach the average gene frequency and there is little advantage in moving from one occupied site to another. If, on the other hand, either number is small the resulting sampling variance maintains a degree of variability between the sites. In this case it becomes 
advantageous to a genotype to distribute its competition more widely through migration.

With this in mind we return now to the more general case having site extinctions, and summarize the major features of our model. First, it is understandable that the ESS migration rate is expected to be large when either there is a high probability of sites becoming extinct (giving immediate individual advantage) or the variance in gene frequency is high due to small numbers of adults or offspring (giving inclusive fitness advantage). Second, allowing for exceptions near $p=0$ (where occurs the "rim" effect already discussed-probably of little biological significance), it is also understandable that ESS migration rate increases as migration is made less perilous (see Figs 2-5).

The effect of introducing sexual reproduction is a decrease (usually slight) in the ESS rate. Introducing stepping-stone migration would also decrease the ESS rate because it reduces the effective variance in gene frequency (i.e. that variance which is perceived by migrants).

Comparison of the ESS and the species optimum (maximum occupancy) migration rate reconciles the results of Roff (1974) and Hamilton \& May (1977). Unless the environmental variance is very small the ESS is constrained to be close to the species optimum. This accounts for the "rims" apparent in Figs 4 and 5 for low migration survival rate, and also for the "trough" apparent in the corresponding position in Fig. 2 (which is present also in principle in Fig. 3 but too narrow to be seen).

This research was carried out at Imperial College Field Station, Silwood Park, Ascot, Berks., U.K. and the numerical results were obtained on Imperial College's CDC computer. HNC was financed by a Natural Environment Research Council grant to the Environmental Management Unit at Silwood Park; RMM was supported in part by NSF grant DEB 79-03290.

\section{REFERENCES}

Comins, H. N. (1980). ESS dispersal in stepping-stone models, in preparation.

Crow, J. F. \& K ImURA, M. (1970). An Introduction to Population Genetics Theory. New York: Harper \& Row.

FELler, W. (1950). An Introduction to Probability Theory and its Applications, Vol. 1. New York: Wiley.

FisHER, R. A. (1930). The Genetical Theory of Natural Selection. Oxford: Oxford University Press.

Hamilton, W. D. (1975). In Biosocial Anthropology (R. Fox, ed.) ASA Studies, London: Malaby Press.

HAMilton, W. D. \& MAY, R. M. (1977). Nature 269,578.

KimuRA, M. \& WeISS, G. H. (1964). Genetics 49,561 .

MaY, R. M. (1974). Stability and Complexity in Model Ecosystems, 2nd edn, Princeton, N.J.: Princeton University Press. 
RofF, D. A. (1975). Oecologia, Berl. 19, 217.

WrIGHT, S. (1969). Evolution and the Genetics of Populations. Chicago: University of Chicago Press.

\section{APPENDIX A}

This appendix outlines the derivation of the evolutionary stable strategy for dispersal probability in the stochastic island model with random extinction. Generating functions are used extensively; all the theorems required are grouped together in Appendix B.

In the classical island model the mean $\rho$ and the variance $S$ of the distribution of numbers of mutants per site suffice to determine the mean and the variance in the next generation. This is almost still true for the present model; however it is now possible for some sites to become empty. Thus a third parameter is required to describe the state of the system, namely the fraction of sites occupied $f$. The three parameters $\rho, \mathrm{S}$ and $f$ suffice to determine the new values $\rho^{\prime}, S^{\prime}$ and $f^{\prime}$ for the next generation.

The expression for the ESS migration probability is found to depend solely on $f$ and $\mathrm{S}$, and not on $\rho$ or any other details of the probability distribution. Thus in calculating the ESS we need only be concerned with $\rho, \mathrm{S}$ and $f(\rho$ is still required, as it affects $S$ ).

The ESS migration probability $v$ has the property that it can coexist stably with a phenotype having slightly different migration probability $v+\varepsilon$. This is a consequence of the fact that $v$ represents an extremum of "gene fitness", which should therefore have zero derivative with respect to $\mathrm{v}$ at this point. We therefore wish to discover what values of $v$ will lead to an equilibrium of $\rho, \mathrm{S}$ and $f$ when the two genotypes have migration probabilities $v$ and $v^{\prime}=v+\varepsilon$.

This appendix derives the conditions for no change between generations in each of the parameters $\rho, \mathrm{S}$ and $f$, given that the other two have fixed values. Taking the limit $\varepsilon \rightarrow 0$, the second and third conditions specify the equilibrium values of $\mathrm{S}$ and $f$, while the first gives a condition on migration rate $v$ which must be satisfied for an equilibrium to exist. Since this condition is independent of gene frequency $\rho$, it gives the value of $v$ which is an ESS with respect to small variations.

Note that although the $\mathrm{S}$ and $f$ equilibria are individually stable if the other two state variables are held constant, it has not been shown that the overall equilibrium is stable. Thus it is conceivable, although unlikely, that $\rho$, $S$ and $f$ execute a complicated limit cycle, with different values of $v$ being optimal at each point. The mathematics required to dismiss this possibility is rather formidable; however some qualitative stability considerations are discussed in the main text. 


\section{Equlibrium 0 ccupation Probability}

The equilibrium of $f$ represents a balance between colonization of empty sites, and the depopulation (by emigration) and extinction (by exogenous forces) of occupied sites. The new value is

$$
f^{\prime}=(1-f)\left(1-p_{\mathrm{ee}}\right)+f\left(1-p_{f e}\right)
$$

where $p_{f e}=$ probability that an occupied site becomes empty, $p_{e e}=$ probability that an empty site remains empty. For $v^{\prime}=v$ and Poisson arrival of immigrants, and arbitrary distribution of offspring with generating function $\mathrm{g}(\mathrm{x})$, it can be shown, using (B1) and (B2), that:

$$
\begin{aligned}
& p_{f e}=X+(1-X) g(v)^{k} \mathrm{e}^{-n v \theta} \\
& p_{e e}=X+(1-X) \mathrm{e}^{-n v \theta}
\end{aligned}
$$

where the quantities $\mathrm{X}, \mathbf{k}, n, v$ and $\boldsymbol{\theta}$ are as defined in Table 1 . If both immigrants and offspring are Poisson distributed then $g(x)=\exp [\tau(x-1)]$, where $\boldsymbol{\tau}=\mathbf{n} / \mathbf{k}$ is the average reproductive rate. Substituting in (Al) and taking the equilibrium $f^{\prime}=f$, we can rearrange to get

$$
p=\theta_{\mathrm{I}} \frac{C+\mathrm{e}^{-n(1-v)} \mathrm{e}^{-n v \theta}}{1-\mathrm{e}^{-n v \theta^{2}}}+1_{\mathrm{I}}
$$

where $\mathrm{C}=\boldsymbol{X} /(1-\boldsymbol{X})$.

It can be shown that the right hand side of (A3) is monotonic increasing with $\theta$. Thus (A3) can be inverted to give $\theta$ as a function of $v, n$ and $\mathrm{p}$. We then obtain $f$ using $f=\theta / p$. The equilibrium of $f$ exists provided

$$
(1-X)\left[n v p+\left(1-\mathrm{e}^{-n(1-v)}\right)\right]>1 .
$$

If it exists the equilibrium is necessarily stable. This can be shown by graphical arguments, given that $\mathrm{f}^{\prime}$ is a monotonic increasing function of $f$.

Note that if $v^{\prime}=v+\varepsilon$ the equilibrium value off contains terms linear in $\varepsilon$; these have been ignored by setting $v^{\prime}=v$ in the above derivation.

\section{Condition for No Change in Gene Frequency}

We first derive the probabilities that a certain number of mutant or wildtype juveniles compete for a site which previously contained $i$ mutant adults and $\mathbf{k}-\boldsymbol{i}$ wildtype adults. The probability of $j$ mutants is given by

$$
M_{j}^{(i)}=\sum J_{r}^{\prime} I_{j-r}^{\prime}
$$

where $I_{j-r}^{\prime}$ is the probability of $j-r$ mutant immigrants, given by a Poisson 
distribution with mean $n \theta v^{\prime} \rho$. $J_{r}^{\prime}$ is the probability that $r$ mutant offspring remain behind:

$$
J_{r}^{\prime}=\sum G_{l}^{(i)} B_{r}^{(l)}\left(1-v^{\prime}\right)
$$

where $B_{r}^{(l)}\left(1-v^{\prime}\right)$ is the probability that $r$ out of $l$ events occur if each has independent probability $1-v^{\prime}$ (i.e. binomial distribution). $G_{l}^{(i)}$ is the probability (same for both genotypes) that $i$ parents have $l$ offspring.

If $\mathrm{g}(\mathrm{x})$ is the generating function of the number of offspring per adult, then the g.f. of $G^{(i)}$ is $[\mathrm{g}(\mathrm{x})]^{\prime}$ (cf. B2), and the g.f. of $J^{\prime}$ is $\left[\boldsymbol{g}\left(\boldsymbol{u}^{\prime}\right)\right]^{i}$ (cf. Bl), where $\boldsymbol{u}^{\prime}$ is the g.f. of the binomial distribution with one trial, that is

$$
u^{\prime}(x)=v^{\prime}+\left(1-v^{\prime}\right) x
$$

Substituting (A7) and the Poisson generating function (B3) into (A5), and using (B4), we obtain the g.f. of $\boldsymbol{M}^{(i)}$ :

$$
m^{(i)}(x)=\left[g\left(u^{\prime}\right)\right]^{i} \mathrm{e}^{n \theta v^{\prime} \rho(x-1)} .
$$

A similar derivation shows that the g.f. of the probability $W_{i}^{(k-i)}$ of $j$ wildtype competitors is:

$$
w^{(k-i)}(x)=[g(u)]^{k-i} \mathrm{e}^{n \theta v(1-\rho)(x-1)} .
$$

Given that the site does not become depopulated, the mean number of mutant adults resulting from fair competition is given by

$$
\begin{aligned}
\left\langle i^{\prime}\right\rangle & =\left[k \sum_{s>0} \sum_{j}(j / s) M_{j}^{(i)} W_{s-j}^{(k-i)}\right] /\left(1-M_{0}^{(i)} W_{0}^{(k-i)}\right) \\
& \equiv k \eta(i) /\left(1-M_{0}^{(i)} W_{0}^{(k-i)}\right) .
\end{aligned}
$$

Note that it was necessary to correct for sites which died out, and thus had no definite value of i'.

A similar expression is obtained for the mean number of mutant adults in newly colonized sites:

$$
\begin{aligned}
\left\langle i^{\prime}\right\rangle & =\left[k \sum_{s>0} \sum_{j}(j / s) I_{j}^{\prime} I_{s-i}\right] /\left(1-I_{0}^{\prime} I_{0}\right) \\
& \equiv k \zeta /\left(1-I_{0}^{\prime} I_{0}\right)
\end{aligned}
$$

where $I_{j}^{\prime}$ and $I_{s-j}$ are respectively the probabilities that there are $j$ mutant and $s-j$ wildtype immigrants. These are Poisson distributed with means $n \theta v^{\prime} \rho$ and $n \theta v(1-\rho)$. 
Suppose that a fraction $\phi_{i}$ of the occupied sites contained $i$ mutants. Then the previous mutant gene frequency was

$$
\rho=\sum \phi_{i}(i / k) \text {. }
$$

Including the appropriate weighting factors the new gene frequency is

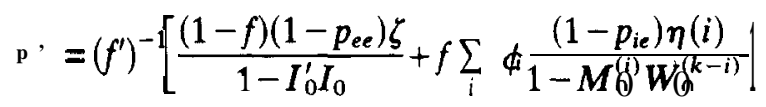

where $p_{e e}=$ probability that an empty site remains empty, $p_{i e}=$ probability that a site with $i$ mutants becomes empty, $f^{\prime}=$ new value of $f$.

Taking account of random (exogenous) extinction we have

$$
\begin{aligned}
& p_{e e}=X+(1-X) I_{0}^{\prime} I_{0} \\
& p_{l e}=X+(1-X) M_{(j)}^{(j)} W^{(k-i)} .
\end{aligned}
$$

Using (B5) and (B3) it can be shown that

$$
\begin{aligned}
\zeta & =\sum_{s>0} \sum_{\mathrm{j}}(j / s) I_{i}^{\prime} I_{s-i} \\
& =\frac{v^{\prime} \rho}{v^{\prime} \rho+v(1-\rho)}\left[1-I_{0}^{\prime} I_{0}\right]
\end{aligned}
$$

Substituting (A14) and (A15) into (A13), and using the equilibrium condition for $f$

$$
\rho^{\prime}=(1-X) \sum_{i} \phi_{i} \eta(i)+\frac{v^{\prime} \rho}{v^{\prime} p+v(1-\rho)}\left[X+(1-X) \sum_{!} \phi_{i} M_{0}^{(i)} W_{0}^{(k-i)}\right] .
$$

The square bracket is just the average probability that an occupied site will become empty. Thus (A16) has the interpretation that the change in gene frequency consists of one term resulting from competition for already occupied sites, and a second term resulting from the division (in the ratio of representation in the migrant pool) of sites which have been allowed for the present to become empty, but which will be recolonized some time in the future.

Using (BS) we have that

$$
\begin{aligned}
\eta(i) & =\sum_{s>0} \sum_{i}(j / s) \boldsymbol{M}_{j}^{(i)} W_{s-j}^{(k-i)} \\
& =\int_{0}^{1} \dot{m}^{(i)}(x) w^{(k-i)}(x) \mathrm{dx}
\end{aligned}
$$


where the dot denotes differentiation. Integrating by parts and substituting into (A16) we get after rearrangement (using B6):

$$
\begin{aligned}
\frac{\rho^{\prime}}{1-\mathrm{x}}= & {\left[1-\frac{v(1-\rho)}{v^{\prime} \rho+v(1-\rho)}\right] C+1-\sum_{i} \phi_{i} \gamma(i) } \\
& -\frac{v(1-\rho)}{v^{\prime} \rho+v(1-\rho)}-\left[\sum \phi_{i} m^{(i)}(0) w^{(k-i)}(0)\right]
\end{aligned}
$$

where

$$
\gamma(i)={ }_{\mathrm{J}_{0}}^{1} m^{(i)}(x) \dot{w}^{(k-i)}(x) \mathrm{dx} .
$$

When $\varepsilon=0$ (i.e. $v^{\prime}=v$ ) it must be that $\rho^{\prime}=\rho$ since the two genes are indistinguishable. If $\varepsilon$ is small but non-zero, a necessary condition for an ESS is that the first order term in $\varepsilon$ must be zero, otherwise $\varepsilon$ could be chosen (either positive or negative) such that $\rho^{\prime}>\rho$. Thus we have the condition

$$
\left[\frac{\partial \boldsymbol{q}}{\partial v^{\prime}}{\underline{v^{\prime}=v}}=0\right.
$$

Substituting (A8) and (A9) in (A18) and differentiating gives the result:

$$
\begin{aligned}
0= & \rho(1-\rho)\left[\frac{C}{v}+\sum_{i} \phi_{i}\left\{\frac{1}{v}+k\left(\tau \theta-\frac{i}{k \rho} \frac{\dot{g}(v)}{g(v)}\right)\right\} g(v)^{k} \mathrm{e}^{-n v \theta}\right] \\
& -\sum_{i} \phi_{i}\left[\partial \gamma(i) / \partial v^{\prime}\right]-(1-\rho) \sum_{i} \frac{\partial \phi_{i}}{\partial v^{\prime}} g(v)^{k} \mathrm{e}^{-n v \theta} \\
& -\sum_{i} \frac{\partial \phi_{i}}{\partial v^{\prime}} \int_{0}^{1}[n \theta v(1-\rho)+(k-i)(1-v) \dot{g}(u) / g(u)] g(u)^{k} \mathrm{e}^{n \theta v(x-1)} \mathrm{d} x .
\end{aligned}
$$

The $\partial \phi_{i} / \partial v^{\prime}$ terms are easily disposed of, since we have (using A20):

$$
\begin{gathered}
\sum_{i} \frac{\partial \phi_{i}}{\partial v^{\prime}}=\frac{\partial}{\partial v^{\prime}} \sum_{i} \phi_{i}=\frac{\partial}{\partial v^{\prime}}(1)=0 \\
\sum_{i} \frac{\partial \phi_{i}}{\partial v^{\prime}}(k-i)=\frac{\partial}{\partial v^{\prime}}(k)-k \frac{\partial}{\partial v^{\prime}} \sum_{i} \phi_{i}\left(\frac{i}{k}\right) \\
=-k \frac{\partial \rho}{\partial v^{\prime}}=0 .
\end{gathered}
$$

The first sum over $i$ in (A21) is easily performed using the definition 
$\rho=\sum_{i} \phi_{i}(i / k)$ :

$$
\begin{aligned}
0= & \rho(1-\rho)\left[\frac{C}{v}+\left\{\frac{1}{v}+k\left(\tau \theta-\frac{\dot{g}(v)}{g(v)}\right)\right\} g(v)^{k} \mathrm{e}^{-n v \theta}\right] \\
& -\sum_{i} \phi_{i} \partial \gamma(i) / \partial v^{\prime} .
\end{aligned}
$$

From (A19) we have

$$
\frac{\partial \gamma(i)}{\mathrm{ad}}=\int_{0}^{1}\left[\frac{\partial m^{(i)}}{\partial v^{\prime}}\right]\left[\frac{\left.\partial w^{(k-i}\right)}{\partial x}\right] \mathrm{d} x .
$$

Calculating the derivatives from (AS) and (A9) and substituting, gives

$$
\left[\frac{\partial \gamma(i)}{\partial v^{\prime}}\right]_{v^{\prime}=v}=\int_{0}^{1} K(y) \mathrm{d} y[-n \theta \rho+i \dot{g} / g][n \theta v(1-\rho)+(k-i)(1-v) \dot{g} / g]
$$

where

$$
\begin{aligned}
y & =1-x \\
\mathrm{~K}(\mathrm{y}) & =y g(u)^{k} \mathrm{e}^{-n \theta v y} \\
u & =1-(1-v) y
\end{aligned}
$$

and $\boldsymbol{g}$ and $\dot{g}$ are evaluated at $u$.

Using the definitions of the mean and variance of $i / k$ :

$$
\begin{aligned}
& \rho=\sum_{i} \phi_{i}(i / k) \\
& S=\sum_{i} \phi_{i}(i / k-\rho)^{2}
\end{aligned}
$$

we can perform the second sum over $i$ in (A22), giving

$$
-\sum \phi_{i}\left[\partial \gamma(i) / \partial v^{\prime}\right]_{v^{\prime}=v}=\rho(1-\rho) T_{1}+S T_{2}
$$

where

$$
\begin{aligned}
& T_{1}=n^{2} \int_{0}^{1} \mathrm{~K}(\mathrm{y}) \mathrm{d} y\left[\theta-\tau^{-1} \dot{g} / g\right]\left[v \theta+(1-v) \tau^{-1} \dot{g} / g\right] \\
& T_{2}=n^{2}(1-v) \int_{0}^{1} K(y) \mathrm{d} y\left[\tau^{-1} \dot{g} / g\right]^{2} .
\end{aligned}
$$

Thus the ESS condition is that the sum of (A26) and the first part of (A22) should be zero. 
For the case of Poisson distributed offspring $\mathrm{g}(\mathrm{x})=\exp [\tau(x-1)]$. Thus $\tau^{-1} \dot{g} / g=1$ and the integrals are easily performed to give

$$
C+\{1-n v(1-\theta)\} Z=[(\alpha / \theta)(1-Z)-n v Z][(1-\theta)-(1-\alpha) F]
$$

where

$$
\alpha=v \theta /[(1 \quad v)+v \theta]
$$

and

$$
Z=\exp [-n\{(1-v)+\theta v\}]
$$

is the probability that an occupied site will become depopulated, and

$$
F=S /[\rho(1-\rho)] .
$$

It will be shown below that the equilibrium value of $\boldsymbol{F}$ is independent of the mutant gene frequency $\rho$. Thus in general $\rho(1-\rho)$ factors out of the ESS condition, so that the ESS is never frequency dependent. Also $S \leq \rho(1-\rho)$ at equilibrium, so $\boldsymbol{F}$ lies between 0 and 1.

3. Equilibrium Variance in Gene Frequency (using $\boldsymbol{v}^{\prime}=\boldsymbol{v}$ ).

In the model there are two benefits of migration; colonization of empty sites, and competition for occupied sites in which the gene frequency is significantly different from the present site. Therefore the variance in gene frequency is an important factor in determining the ESS migration rate, which is higher if the variance is high. Note that in a stepping-stone model the variance is effectively smaller, due to correlations between neighbouring sites, so the ESS migration rate would be somewhat smaller.

Let $P_{r}$ be the probability that a previously occupied site is still occupied and has $r$ mutants:

$$
P_{r}=(1-X) \sum \phi_{i} \sum_{s>0} B_{r}^{(k)}(j / s) M_{j}^{(i)} W_{s-j}^{(k-i)}
$$

and let $\boldsymbol{P}_{\boldsymbol{N}_{r}}$ be the probability that a previously empty site is occupied and has $r$ mutants:

$$
P_{N r}=(1-X) \sum_{s>0} B_{r}^{(k)}(j / s) I_{j}^{\prime} I_{s-j} .
$$

Then the overall probability that a site is both occupied and has $r$ mutants is 
$f P_{r}+(1-f) P_{N r}$; at equilibrium the probability that an occupied site has $r$ mutants is

$$
\boldsymbol{\phi}_{r}^{\prime}=P_{r}+[(1-f) / f] P_{N r} .
$$

In order to derive the new variance $S$ ' we calculate the moment generating function of this distribution:

$$
\Phi^{\prime}(t)=(1-X) \sum_{\substack{s>0 \\ i}} \sum_{r} B_{r}^{(k)}(j / s) \mathrm{e}^{r t} R_{s j}
$$

where

$$
R_{s j}=\sum_{i} \phi_{i} M_{j}^{(i)} W_{s-j}^{(k-i)}+\{(1-f) / f\} I_{j}^{\prime} I_{s-j}
$$

Using the binomial theorem

$$
\sum_{r} B_{r}^{(k)}(j / s) \mathrm{e}^{t}=\left(\frac{s-j}{j}+\frac{j e^{t}}{s}\right)^{k} .
$$

Substituting this, and expanding in powers of $\boldsymbol{t}$, we get (using B7):

$$
\begin{gathered}
\rho^{\prime}=(1-X) \sum_{\substack{s>0 \\
j}}(j / s) R_{s j} \\
S^{\prime}+\left(\rho^{\prime}\right)^{2}=(1-X) \sum_{s>0}\left\{(j / s)-\frac{k-1}{k} \frac{(s-j) j}{s^{2}}\right\} R_{s j} .
\end{gathered}
$$

The first equation is equivalent to ( $\mathrm{Al} 3)$ and at equilibrium we should have $\boldsymbol{\rho}^{\prime}=\boldsymbol{\rho}$. Substituting this in the second equation gives

$$
S^{\prime}=\rho(1-\rho)-(1-X) \frac{k-1}{k} \sum_{s>0} \frac{(s-j) j}{s^{2}} g_{j}
$$

Thus

$$
S^{\prime}=\rho(1-\rho)-(1-X) \frac{k-1}{k}\left[\sum_{i} \phi_{i} Q_{i}+\{(1-f) / f\} Q_{N}\right]
$$

where

$$
\begin{aligned}
Q_{i} & =\sum_{s>1} \sum_{i} \frac{(s-j) j}{s^{2}} M_{j}^{(i)} W_{s-i}^{(k-i)} \\
Q_{N} & =\sum_{s>1} \sum_{i} \frac{(s-j) j}{s^{2}} I_{j}^{\prime} I_{s-j}
\end{aligned}
$$


Using (B8) and the generating functions (A8) and (A9) gives

$Q_{i}=\int_{0}^{1} \frac{\mathrm{d} y}{y} \int_{0}^{y} \mathrm{~d} x L(x)[n v \theta \rho+i(\dot{g} / g)(1-v)][n v \theta(1-\rho)+(k-i)(\dot{g} / g)(1-v)]$

where

$$
\begin{aligned}
u & =v+(1-v) x \\
L(x) & =g(u)^{k} \mathrm{e}^{n v \theta(x-1)} x .
\end{aligned}
$$

As with (A22) this simplifies when summed over $i$ :

$$
\sum \phi_{i} Q_{i}=\rho(1-\rho) A_{1}-S A_{2}
$$

where

$$
\begin{aligned}
& A_{1}=n^{2} \int_{0}^{1} \frac{\mathrm{d} y}{y} \int_{0}^{y}\left[v \theta+(1-v) \tau^{-1} \dot{g} / g\right]^{2} L(x) \mathrm{dx} \\
& A_{2}=n^{2}(1-v)^{2} \int_{0}^{1} \frac{\mathrm{d} y}{y} \int_{0}^{y}\left[\tau^{-1} \dot{g} / g\right]^{2} L(x) \mathrm{d} x .
\end{aligned}
$$

A similar process leads to

$$
Q_{N}=\rho(1-\rho) A_{3}
$$

where

$$
A_{3}=n^{2}(v \theta)^{2} \int_{0}^{1} \frac{\mathrm{d} y}{y} \int_{0}^{y} \mathrm{e}^{n v \theta(x-1)} x \mathrm{~d} x .
$$

We define the function

$$
\begin{aligned}
H(a) & =a^{2} \int_{0}^{1} \frac{\mathrm{d} y}{y} \int_{0}^{y} x \mathrm{~d} x \mathrm{e}^{a(x-1)} \\
& =1-e^{-a}[1+E i(a)-\ln a-\gamma]
\end{aligned}
$$

where y is Eulers' constant $(\mathrm{O}-57721 \ldots)$ and $E i$ is a standard exponential integral. $H(a)$ is sigmoid in form, and ranges from 0 to 1 for $0 \leq a<\infty$. Then (A38) becomes

$$
F^{\prime}=1-(1-X) \frac{k-1}{k}\left[A_{1}-F A_{2}+\{(1-f) / f\} H(n v \theta)\right]
$$


where $\boldsymbol{F}=\boldsymbol{S} /[\boldsymbol{\rho}(1-\rho)], \boldsymbol{F}^{\prime}=\boldsymbol{S}^{\prime} /[\boldsymbol{\rho}(1-\mathrm{p})]$. At equilibrium we have $\boldsymbol{F}^{\prime}=\boldsymbol{F}$, so

$$
\boldsymbol{F}=\frac{1-(1-X) \frac{k-1}{k}\left[A_{1}+\{(1-f) / f\} H(n v \theta)\right]}{1-(1-X) \frac{\mathrm{k}-\mathrm{l}}{k} A_{2}}
$$

Since $\dot{g} / g>0$, we have $A_{1}>A_{2}$, so $F \leq 1$.

For Poisson distributed offspring $\mathrm{g} / \mathrm{g}=\boldsymbol{\tau}$ and the integrals can be evaluated to give

$$
\boldsymbol{F}=\frac{1-(1-X) \frac{k-1}{k}[H(n v \theta / \alpha)+\{(1-f) / f\} H(n v \theta)]}{1-(1-X) \frac{k-1}{k}(1-\alpha)^{2} H(n v \theta / \alpha)}
$$

where as before $\alpha=v \theta /[(1-v)+v \theta]$. Thus the equilibrium conditions for an ESS consist of (A3), (A29) and (A47).

\section{A Special Case}

We will now demonstrate that the ESS condition reduces to Hamilton and May's (1977) equation 3 in the special case $\boldsymbol{k}=1, \mathrm{X}=0$. For this case (A47) gives $\boldsymbol{F}=1$, and hence (A29) becomes

$$
\{1-n v(1-\theta)\} Z=[(\alpha / \theta)(1-Z)-n v Z](\alpha-\theta) .
$$

Solving this as a linear equation in $\mathrm{Z}$ and simplifying the denominator we get

$$
\mathrm{Z}=(\alpha-\theta) /\left[\alpha+\theta\left(\alpha^{-1}-1\right)(1-n v)\right] .
$$

Substituting $\alpha=v \theta /\left[\left(\begin{array}{ll}1 & v\end{array}\right)+v \theta\right]$ and multiplying both top and bottom by $v(1-v+v \theta)$

$$
Z=\frac{v^{2} \theta-v \theta(1-v+v \theta)}{v^{2} \theta+(1-v)(1-v+v \theta)(1-n v)}
$$

Substituting the definitions $\mathrm{Z}=\exp [-n(1-v+v \theta)]$ and $\theta=p f$ we obtain

$$
\exp [-n(1-v+v p f)]=\frac{v^{2} p f-v p f(1-v+v p f)}{v^{2} p f+(1-v)(1-v+v p f)(1-n v)}
$$

This is equivalent to Hamilton and May's equation, disregarding several misprints in their published version. 


\section{APPENDIX B}

The following theorems for generating functions are used in Appendix A. These are standard theorems (Feller, 1950) except for (B5) and (B8).

(B1) If $N$ is the sum of $n$ variates chosen independently from the distribution $\left\{\boldsymbol{A}_{i}\right\}$ then the generating function $c(x)$ of the distribution of $N$ is (Fisher, 1930)

$$
c(x)=f(a(x))
$$

where $\mathrm{f}(\mathrm{x})$ is the $\mathrm{g.f}$. of the distribution of the number of samples $n$, and $\mathrm{U}(\mathrm{X})$ is the g.f. of $\left\{\boldsymbol{A}_{\boldsymbol{i}}\right\}$.

(B2) A particular case of (B1). If the number of samples $n$ is constant then

$$
c(x)=[a(x)]^{n}
$$

(B3) The Poisson distribution with mean $\tau$ has generating function

$$
f(x)=e^{\tau(x-1)}
$$

(B4) If a(x) is the g.f. Of $\left\{\boldsymbol{A}_{\boldsymbol{i}}\right\}$ and $\mathrm{b}(\mathrm{x})$ is the g.f. of $\left\{\boldsymbol{B}_{\boldsymbol{i}}\right\}$, then $\boldsymbol{c}(\boldsymbol{x})=\boldsymbol{a}(\boldsymbol{x}) \boldsymbol{b}(\boldsymbol{x})$ is the g.f. of the distribution $\left\{C_{i}\right\}$, where

$$
C_{i}=\sum_{j} A_{i} B_{j-i}
$$

(B5) If $a(x)$ is the g.f. of $\left\{\boldsymbol{A}_{\boldsymbol{i}}\right\}$ and $\boldsymbol{b}(\boldsymbol{x})$ is the g.f. of $\left\{\boldsymbol{B}_{\boldsymbol{i}}\right\}$ and

$$
P=\sum_{\substack{s, l \\(s>0)}} A_{j} B_{s-j} j / s
$$

then

$$
\boldsymbol{P}={ }_{\mathrm{I}_{0}}^{1}(\mathrm{~d} a / \mathrm{d} x) b(x) \mathrm{dx}
$$

This can be proved using projection operators, which are defined as follows:

$$
\hat{T}\left(\sum_{i} a_{i} x^{i}\right)=a_{i} x^{i}
$$

(B6) If $a(x)$ is the g.f. of $\left\{A_{i}\right\}$ then $a(0)=A_{0}$ and $a(1)=1$.

(B7) If $a(x)$ is the g.f. of $\left\{A_{i}\right\}$ then

$$
\begin{aligned}
\Phi(t) & =a\left(e^{t}\right) \\
& =\mu_{1}+\frac{\mu_{2} t^{2}}{2 !}+\frac{\mu_{3} t^{3}}{3 !}+\ldots
\end{aligned}
$$

where $\boldsymbol{\mu}_{i}$ is the ith moment of $\left\{\boldsymbol{A}_{i}\right\}$ about zero. 
(B8) If a(x) is the g.f. of $\left\{\boldsymbol{A}_{\boldsymbol{i}}\right\}$ and $\mathrm{b}(\mathrm{x})$ is the g.f. of $\left\{\boldsymbol{B}_{i}\right\}$ and

$$
Q=\sum_{\substack{s, j \\(s>1)}} A_{j} B_{s-j} j(s-j) / s^{2}
$$

then

$$
Q=\int_{0}^{1} \frac{\mathrm{d} y}{y} \int_{0}^{1}(\mathrm{~d} a / \mathrm{d} x)(\mathrm{d} b / \mathrm{d} x) x \mathrm{~d} x
$$

This can be proved using projection operators in a similar manner to (B5).

\section{APPENDIX C}

The analogue of Appendix A for sexual reproduction is most readily approached by considering the $2 \mathrm{k}$ genes in the gene pool at each site, rather than the $k$ adult organisms. In the full model a number of serious complications arise; for example all migration involves pairs of genes, and there are correlations between the numbers of immigrant genes of different types. We will therefore only consider the limiting case where the number of offspring per site is extremely large. For asexual reproduction (and provided $v \neq 1$, $p \neq 0)(\mathrm{A} 3),(\mathrm{A} 29)$ and (A47) reduce in this limit to:

$$
\begin{aligned}
& f=1-X \\
& C=(\alpha / \theta)[(1-\theta)-(1-\alpha) F] \\
& F=\left[k-(k-1)(1-X)(1-\alpha)^{2}\right]^{-1}
\end{aligned}
$$

Suppose that reproduction is sexual, and that there are two genotypes; wildtype with migration probability $v$, and mutant with migration probability $v+\varepsilon$. Heterozygotes are supposed to have migration probability $\mathrm{v}+\mathrm{E} / 2$.

The expected numbers of wildtype and mutant immigrant genes at a site are respectively (Crow and Kimura, 1970):

$$
\begin{gathered}
W: \quad k f p \sum_{i} \phi_{i}\left\{2(1-i / 2 k)^{2} v+2(i / 2 k)(1-i / 2 k)(v+\varepsilon / 2)\right\} \\
=k \theta\{2 v(1-\rho)+\varepsilon(\rho(1-\rho)-S)\}
\end{gathered}
$$

$\mathrm{M}: \quad k p f \sum_{i} \phi_{i}\left\{2(i / 2 k)^{2}(v+\varepsilon)+2(i / 2 k)(1-i / 2 k)(v+\varepsilon / 2)\right\}$

$$
=k \theta\{2 v \rho+\varepsilon(\rho(1+\rho)+S)\}
$$


where

$$
\begin{aligned}
& \rho=\sum(i / 2 k) \phi_{i} \\
& S=\sum(i / 2 k-\rho)^{2} \phi_{i} .
\end{aligned}
$$

The expected numbers of sedentary competitors (genes) at a site which previously had $\mathbf{i}$ out of $\mathbf{2 k}$ mutant genes are

$$
\begin{array}{lll}
\text { W } & : & k\left[2(1-i / 2 k)^{2}(1-v)+2(i / 2 k)(1-i / 2 k)(1-v-\varepsilon / 2)\right] \\
\text { M } & : \quad k\left[2(i / 2 k)^{2}(1-v-\varepsilon)+2(i / 2 k)(1-i / 2 k)(1-v-\varepsilon / 2)\right]
\end{array}
$$

Since the number of offspring is extremely large the probability of any gene in the next generation being a mutant is given by the ratio of the expected total number of mutant genes competing to the expected total of both types. Letting $\boldsymbol{\eta}=i / 2 k$ be the previous fraction of mutants and $\eta^{\prime}$ the expected new fraction, we obtain

where

$$
\eta^{\prime}=(\mathrm{A}+B \varepsilon) /(E+D \varepsilon)
$$

$$
\begin{aligned}
& A=(1-v) \eta+\theta v \rho \\
& B=\frac{1}{2}[-\eta(1+\eta)+\theta\{S+\rho(1+\rho)\}] \\
& E=(1-v)+v \theta \\
& D=-\eta+\theta \rho
\end{aligned}
$$

To first order in $\varepsilon$ this is

$$
\eta^{\prime}=A / E+\varepsilon[B E-A D] / E^{2}
$$

Note that the denominator $\mathbf{E}$ is independent of $\boldsymbol{\eta}$. Thus to obtain the average value of $\boldsymbol{\eta}^{\prime}$ for all previously occupied sites we sum (C5) over $\mathbf{i}$ to get

where

$$
\left\langle\eta^{\prime}\right\rangle_{\mathrm{OCC}}=\rho+\varepsilon G /[(1-v)+\theta v]
$$

$$
G=S\left\{(1-\alpha)-\frac{1}{2}(1-\theta)\right\}-\frac{1}{2}(1-\theta) \rho(1-\rho)
$$

For previously empty sites we obtain the analogous result

$$
\left\langle\eta^{\prime}\right\rangle_{\mathrm{EMPTY}}=\rho+\varepsilon[S+\rho(1-\rho)] / 2 v
$$

The ESS condition is that $(1-\mathrm{X})$ times the $\varepsilon$ term in (C6) plus X times the $\varepsilon$ term in (C7) should be zero. This can be rewritten as

$$
F=\frac{\{1-\theta(1+C / \alpha)\}}{2(1-\alpha)-\{1-\theta(1+C / \alpha)\}}
$$


where

$$
\begin{aligned}
& C=X /(1-X) \\
& F=S /\{\rho(1-\rho)\}
\end{aligned}
$$

As in the asexual case we require the equilibrium genetic variance $S$ (calculated for $\varepsilon=0$ ). We observe that the first term in (C5) is

$$
\begin{aligned}
\eta^{\prime} & =A / E=(1-\alpha) \eta+\alpha \rho \\
& =\eta+\alpha(\rho-\eta)
\end{aligned}
$$

This formula, together with the number of genes selected $(2 k)$ and $\left\langle\eta^{\prime}\right\rangle_{\text {EMPTY }}=\rho$ from $(\mathrm{C} 7)$, determines the binomial choice of the next generation gene pool, and thus the genetic variance of the next generation. However it is found that the identical formulae are obtained for the asexual case with $2 k$ adults per site in the limit $n \rightarrow \infty$, so the sexual genetic variance is simply given by using $2 k$ instead of $k$ in the third of equations $(\mathrm{Cl})$ :

$$
F=\left[2 k-(2 k-1)(1-X)(1-\alpha)^{2}\right]^{-1}
$$

Thus the ESS is given by (C8) and (C10).

If we define $z=k+\frac{1}{2}$, then the ESS condition can be written

$$
1-\alpha=\{1-\theta(1+C / \alpha)\}\left[z-(z-1)(1-X)(1-\alpha)^{2}\right]
$$

Except for the substitution $k \rightarrow z$ this equation is equivalent to $(\mathrm{Cl})$. Thus, in the $n \rightarrow \infty$ limit, the effect of sexual reproduction is formally equivalent to an increase of $\frac{1}{2}$ in the number of adults in each site. 